\title{
Estado nutricional e a qualidade de vida em homens idosos vivendo em instituição de longa permanência em Curitiba, PR
}

Nutritional status and quality of life in elderly living in long-term care facility in

Curitiba, state of Paraná

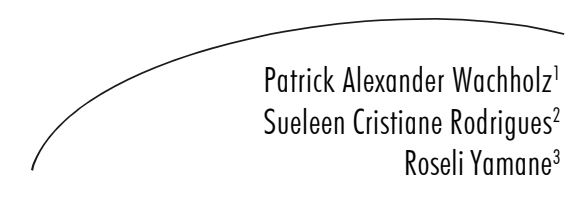

Resumo

Introdução: Com o envelhecimento, o perfil nutricional pode sofrer forte influência de fatores ambientais, biológicos e sociais, que interferem na ingestão alimentar e no aproveitamento dos nutrientes. Estas modificações podem impactar (positiva ou negativamente) as percepções de qualidade de vida. Objetivos: analisar a correlação entre a percepção de qualidade de vida e o estado nutricional em homens idosos institucionalizados em uma unidade de longa permanência, no município de Curitiba-PR. Metodologia: Estudo observacional transversal, baseado na aplicação do Inquérito WHOQOL-bref em amostra de 35 idosos, avaliando as correlações das médias dos escores dos domínios e questões gerais do instrumento com variáveis sociodemográficas e do estado nutricional dos idosos segundo três diferentes métodos. Resultados: $\mathrm{O}$ estado nutricional da amostra segundo o IMC encontrou que 42,8\% dos idosos estão com baixo-peso, $34,3 \%$ são eutróficos, $8,6 \%$ sobrepesos e 14,3\% estão com obesidade. Melhores percepções de qualidade de vida correlacionaram-se com estados de sobrepeso segundo o IMC e PCT, e com idades mais jovens. Conclusões: Os idosos estudados nesta amostra que demonstraram melhores médias nos domínios que compõem o WHOQOL-bref foram os portadores de sobrepesos e menor idade.

\section{Abstract}

Introduction: With aging, nutritional profile can undergo strong influence of environment factors and biological and social changes which intervene in food intake and the good use of nutrients. These changes could impact (positively or negatively) the quality of life perceptions in the aged. Objectives: To analyze the

\footnotetext{
Cursos de Pós-Graduação em Geriatria e em Gerontologia Clinica e Social. Universidade Positivo. Curitiba, PR, Brasil.

2 Escola de Saúde, Curso de Nutrição. Faculdades Integradas do Brasil. Curitiba, PR, Brasil.

3 Curso de Pós-Graduação em Gerontologia Social. Universidade Positivo. Curitiba, PR, Brasil.

Palavras-chave: Idoso. Qualidade de Vida. Estado Nutricional. Saúde do Idoso. 
correlation of the quality of life perceptions and the nutritional status of elderly men institutionalized in a long-term facility in Curitiba city, state of Paraná, Brazil. Methods: Observational survey using transversal methodology. WHOQOLbref inquiry was applied to a selected sample of 35 elderly men, and the correlations of the medians of the domains and general questions from the instrument were analyzed with socio-demographic variables and the nutritional status of the sample, using three different nutritional methods. Results: The nutritional status of the sample according to body mass index (BMI) found $42.8 \%$ of the elderly with low weight, $34.3 \%$ of them eutrophic, $8.6 \%$ with overweight and $14.3 \%$ classified as obese. Better quality of life perceptions were found to be correlated to overweight nutritional status, according to BMI and tricipital skin fold nutritional status index, and being younger. Conclusions: The overweight and the younger elderly from the studied aged sample showed the higher medians in the domains that compose the WHOQOL-bref inquiry.
Key words: Aged. Quality of Life. Nutritional Status. Health of the Elderly.

\section{INTRODUÇÃO}

O envelhecimento afeta diretamente o estado nutricional de um indivíduo, seja por questões senis (patológicas) ou senescentes (fisiológicas). Diversas alterações que ocorrem naturalmente no organismo que envelhece são capazes de modificar o estado nutricional, incluindo desde mudanças nas fases iniciais do processo de alimentação (falha na mastigação, pela ausência de elementos dentários ou diminuição da secreção salivar, por exemplo), até prejuízos ou redução na eficiência dos processos avançados de absorção e eliminação. ${ }^{1-3}$

Não obstante, a senescência promove alterações (hormonalmente induzidas ou não) que contribuem para mudanças nos perfis antropométricos desta população, como modificações nos compartimentos de gordura corporal, diminuição da massa muscular magra (sarcopenia), reduções na estatura, pelo comprometimento da densidade mineral óssea vertebral, e redução do percentual de água corporal, dentre outras. ${ }^{2-6}$

O reconhecimento e a instituição de intervenções precoces nas anormalidades do estado nutricional no idoso, além de se associarem a menores taxas de morbimortalidade, ${ }^{2,7}$ têm exibido benefício adicional, ao melhorar o grau de satisfação de idosos com a própria saúde, bem como com percepções positivas de qualidade de vida. ${ }^{8}$
O construto qualidade de vida é um conceito genérico que procede da interpretação individual do bem-estar subjetivo, e muitas vezes independe das condições de vida e percepções técnicas de saúde intacta, associando-se mais a satisfação com a própria vida e com os valores pessoais e culturais de um indivíduo. Segundo Vecchia, ${ }^{9}$ existem vários aspectos já estudados que podem estar associados à qualidade de vida, como a capacidade funcional, o nível socioeconômico, o estado emocional, a interação social, a atividade intelectual, a capacidade de manutenção de independência para o autocuidado, o suporte familiar, o próprio estado de saúde, os valores culturais, éticos e a religiosidade, o estilo de vida, a satisfação com o emprego e/ou com atividades diárias e o ambiente em que se vive.

Com o propósito de orientar políticas públicas para um envelhecimento bem-sucedido e embasar a formação do profissional de saúde que lida com este segmento etário, parece imprescindível conhecer o que esses idosos efetivamente correlacionam ao seu bem-estar, à felicidade e à realização pessoal - enfim, à boa qualidade de vida. ${ }^{10,11}$

Embora ainda sejam incipientes os estudos que relacionam qualidade de vida e o estado nutricional de idosos, existem evidências científicas que sugerem a contribuição deste fator para saúde e, indiretamente, para a qualidade de vida. ${ }^{2,8,12} \mathrm{O}$ objetivo deste trabalho é analisar a 
correlação entre a percepção de qualidade de vida e o estado nutricional em homens idosos institucionalizados.

\section{METODOLOGIA}

O estudo é do tipo quantitativo observacional, de desenho transversal e características descritivas e analíticas. Foi desenvolvido em uma instituição geriátrica filantrópica, sediada no município de Curitiba - PR, destinada ao abrigo exclusivamente de homens, em sua maioria portadores de insuficiência familiar e/ou financeira.

Foram convidados a participar do estudo os idosos residentes na instituição há no mínimo 12 meses, com idade igual ou superior a 60 anos e que apresentassem cognição preservada, necessária para a compreensão das instruções contidas no termo de consentimento informado e para o preenchimento (individual ou assistido) do instrumento de avaliação da qualidade de vida. Foram excluídos os idosos com diagnóstico atual de depressão maior ou outros transtornos psiquiátricos do Eixo II do DSM IV, bem como internos com comprometimento sensorial (auditivo, visual ou da fala) que impossibilitasse a realização da entrevista.

As variáveis que compuseram parte do elenco de informações analisadas foram obtidas a partir dos dados disponíveis no prontuário único multiprofissional dos idosos, que dentre diversas informações contém os registros dos dados antropométricos dos últimos cinco anos, os diagnósticos clínicos, diagnóstico da atual capacidade funcional (pelo índice de independência na execução de atividades básicas de vida diária - Índice de KATZ") e o diagnóstico cognitivo, reforçado pelo escore da aplicação anual do Mini-Exame de Estado Mental de Folstein $\left(\mathrm{MEEM}^{\dagger}\right)$, além do tempo de institucionalização do idoso.
Os dados antropométricos atualizados, coletados no período do estudo, constituíramse de: peso aferido em quilos (kg), altura aferida em metros (m), a prega cutânea tricipital (PCT) em milímetros $(\mathrm{mm})$, prega cutânea subescapular (PCSE) em milímetros (mm), a circunferência do braço $(\mathrm{CB})$ em centímetros $(\mathrm{cm})$, altura do joelho (AJ) em centímetros $(\mathrm{cm})$ e circunferência da panturrilha (CP) em centímetros $(\mathrm{cm})$.

O peso foi mensurado a partir de uma balança antropométrica da marca Filizola ${ }^{\circledR}$, com capacidade de $150 \mathrm{~kg}$ e precisão de $100 \mathrm{~g}$, com o idoso descalço e usando roupas leves. A estatura foi verificada utilizando-se um estadiômetro vertical fixo à balança. As circunferências foram verificadas com o auxílio de uma fita métrica inelástica com precisão de $1 \mathrm{~mm}$ e as pregas cutâneas através de calibrador da marca Cescorf ${ }^{\circledR}$.

Para os idosos impossibilitados de se aferir a altura e o peso, os dados foram estimados a partir das equações de Chumlea: ${ }^{13}$

ESTATURA ESTIMADA: Homem $=[64,19$ - $(0,04 \times$ idade $)+(2,02 \times$ AJ $)]$ PESO ESTIMADO: Homem $=[0,98 \times \mathrm{CP})+(1,16 \mathrm{x}$ $\mathrm{AJ})+(1,73 \times \mathrm{CB})+(0,37 \times \mathrm{PCSE})-81,69]$

Através do peso (P) e altura (A) de acordo com Chumlea, ${ }^{13}$ diagnosticou-se o estado nutricional (EN) através do índice de massa corporal (IMC $=P / A^{2}$ ), classificado pelos pontos de corte recomendados pela Organização Pan-Americana de Saúde (OPAS), a saber: baixo-peso (IMC $\leq$ $23 \mathrm{~kg} / \mathrm{m}^{2}$ ), eutrofia $\left(\right.$ IMC $>23 \mathrm{e}<28 \mathrm{~kg} / \mathrm{m}^{2}$ ), pré-obesidade $\left(\mathrm{IMC} \geq 28\right.$ e $<30 \mathrm{~kg} / \mathrm{m}^{2}$ ) e obesidade (IMC $\geq 30 \mathrm{~kg} / \mathrm{m}^{2}$ ). Através dos dados da PCT e PCS e CB, foi estimada a quantidade total de gordura subcutânea corporal, segundo a fórmula de Chumlea. ${ }^{14}$

KATZ S et al. Studies of illness in the aged. The index of ADL: a standardized measure of biological and psychological function. J Am Med Assoc. 1963;18(12):914-19.

Folstein MF, Folstein SE, McHugh PR. Mini-mental state: a practical method for grading the cognitive state of patients for the clinician. J Psychiatric Res. 1975; 12:189-98. 
A PCT foi utilizada para a classificação do estado nutricional segundo a tabela de percentis da NHANES III ${ }^{\ddagger}$, adequada pela equação PCT $(\%)=$ PCT obtida $(\mathrm{mm}) /$ PCT percentil $50 \times 100$, e classificada de acordo com Blackburn \& Thornton. ${ }^{15}$
Através da $\mathrm{CB}$, classificou-se o EN segundo a tabela de percentis da NHANES I (1971-1974) demonstrada por Frisancho, ${ }^{16}$ adequada pela equação $C B(\%)=C B$ obtida $(\mathrm{cm}) / C B$ percentil $50 x$ 100. A tabela 1 apresenta os pontos de corte propostos pelos autore ${ }^{15}$ para o EN segundo a PCT e a CB.

Tabela 1 - Classificação do Estado Nutricional segundo a Prega Cutânea Tricipital e a Circunferência do Braço. Curitiba, PR, 2009.

\begin{tabular}{lcccccc}
\hline & $\begin{array}{c}\text { Desnutrição } \\
\text { Grave }\end{array}$ & $\begin{array}{c}\text { Desnutrição } \\
\text { Moderada }\end{array}$ & Desnutriçåave & Eutrofia & Sobrepeso & Obesidade \\
\hline Percentual & $<70 \%$ & 70 a $80 \%$ & 80 a $90 \%$ & 90 a $110 \%$ & 110 a $120 \%$ & $>120 \%$ \\
\hline
\end{tabular}

Fonte: Adaptado de Blackburn GL, Thornton $\mathrm{PA}^{14}$

A qualidade de vida (QV) dos idosos foi analisada a partir da aplicação do inquérito WHOQOL-bref, instrumento desenvolvido pela Organização Mundial da Saúde (OMS), que consiste em 26 questões, sendo duas gerais de qualidade de vida e as demais representando cada uma das 24 facetas que compõem o instrumento original, denominado WHOQOL-100. ${ }^{11}$

A interpretação obedece à sintaxe proposta pelo grupo WHOQOL, ${ }^{17}$ e a pontuação analisada envolverá o escore 0-100 na análise dos domínios e 4-20 na análise das questões 1 e 2 (Q1 e Q2). Quanto maior a pontuação, melhor a percepção de QV. Por consenso e definição, não existem pontos de corte acima dos quais a percepção possa ser interpretada como boa ou ruim; o resultado deve ser sempre interpretado segundo as médias dentro da própria amostra. A confiabilidade do instrumento na amostra foi mensurada através do teste de alfa de Cronbach, considerando aceitáveis valores superiores a 0,5 .

A correlação entre as variáveis antropométricas, estado nutricional e percepções de qualidade de vida foi analisada mediante a aplicação de testes não paramétricos (Qui-quadrado), da análise de variância das médias entre os grupos (ANOVA) e de correlação (Pearson e Spearman), adotando como significativos valores de $\mathrm{p} \leq 0,05$, com intervalo de confiança de $95 \%$.

Este trabalho foi aprovado pelo Comitê de Ética em Pesquisa das Faculdades Integradas do Brasil (protocolo de pesquisa $n^{0}$ 001/2009), e respeitou os princípios éticos contidos na Declaração de Helsinki, além de obedecer aos preceitos contidos na legislação nacional pertinente. Os idosos, antes de convidados, foram orientados sobre o estudo, e os que concordaram em participar assinaram o termo de consentimento livre e esclarecido.

\section{RESULTADOS}

A população total de residentes na instituição no período do estudo era de 88 idosos. Destes, 35 idosos $(39,79 \%)$ preencheram os critérios de inclusão e exclusão propostos pelo estudo. A idade média da amostra foi de 72 (d.p. = 7,77) anos, o mais longevo dos residentes com 92 anos. Destes, 15 (42,9\%) tinham idades entre 60 e 69 anos, 12 $(34,3 \%)$ entre 70 e 79 anos, e oito (22,9\%) tinham 
80 anos ou mais. A escolaridade média não ultrapassou 3,71 anos completos de estudo formal, e o tempo médio de institucionalização foi de $7,55$ (d.p. $=7,54)$ anos.

Através da interpretação do Índice de Katz, diagnosticou-se que 29 (82,85\%) dos 35 idosos eram independentes, quatro $(11,42 \%)$ eram dependentes totais e dois $(5,71 \%)$ eram dependentes parciais para a execução de atividades básicas de vida diária.

A pontuação média da amostra segundo o escore obtido pela aplicação do MEEM foi de 23 pontos (escore 0-30). Tendo em vista a baixa escolaridade da amostra, de acordo com Almeida, ${ }^{18}$ este resultado seria suficiente para sugerir que os idosos não possuíam diagnóstico de demência. Antes da admissão no estudo, porém, o diagnóstico negativo para a condição foi confirmado pela revisão do prontuário médico, sendo excluídos os idosos portadores de síndromes demenciais, independentemente da etiologia e severidade destas.

A análise do estado nutricional $(\mathrm{EN}) \mathrm{da}$ amostra, adotando os critérios do IMC, demonstrou que $42,8 \%$ dos idosos estavam com baixo-peso, $34,3 \%$ dos idosos eram eutróficos, $8,6 \%$ encontravam-se pré-obesos e $14,3 \%$ estavam com obesidade. A tabela 2 apresenta a distribuição do EN segundo o IMC, categorizado segundo os três grupos de diferentes faixas etárias dentro da amostra.

Tabela 2 - Classificação do Estado Nutricional segundo o Índice de Massa Corporal e a faixa etária da amostra, em percentual. Curitiba, PR, 2009.

\begin{tabular}{lcccc}
\hline \multicolumn{1}{c}{ Faixa etária } & Baixo peso & Eutrofia & Préobesidade & Obesidade \\
\hline 60 a 69 anos & 33,3 & 26,6 & 20 & 20 \\
70 a 79 anos & 75 & 25 & 0 & 0 \\
80 anos ou mais & 12,5 & 62,5 & 0 & 25 \\
\hline
\end{tabular}

A análise da classificação do EN obtido pela CB e pela PCT (figura 1) identificou diferenças significativas dentro da amostra, com proporções significativamente superiores de sobrepeso e obesidade segundo a PCT, quando comparada com o EN segundo a CB. A última, pois, identificou maior proporção de idosos com eutrofia e desnutrição moderada/leve. Segundo a CB, porém, poucos idosos seriam identificados como sobrepeso e nenhum como desnutrido grave e obeso. 


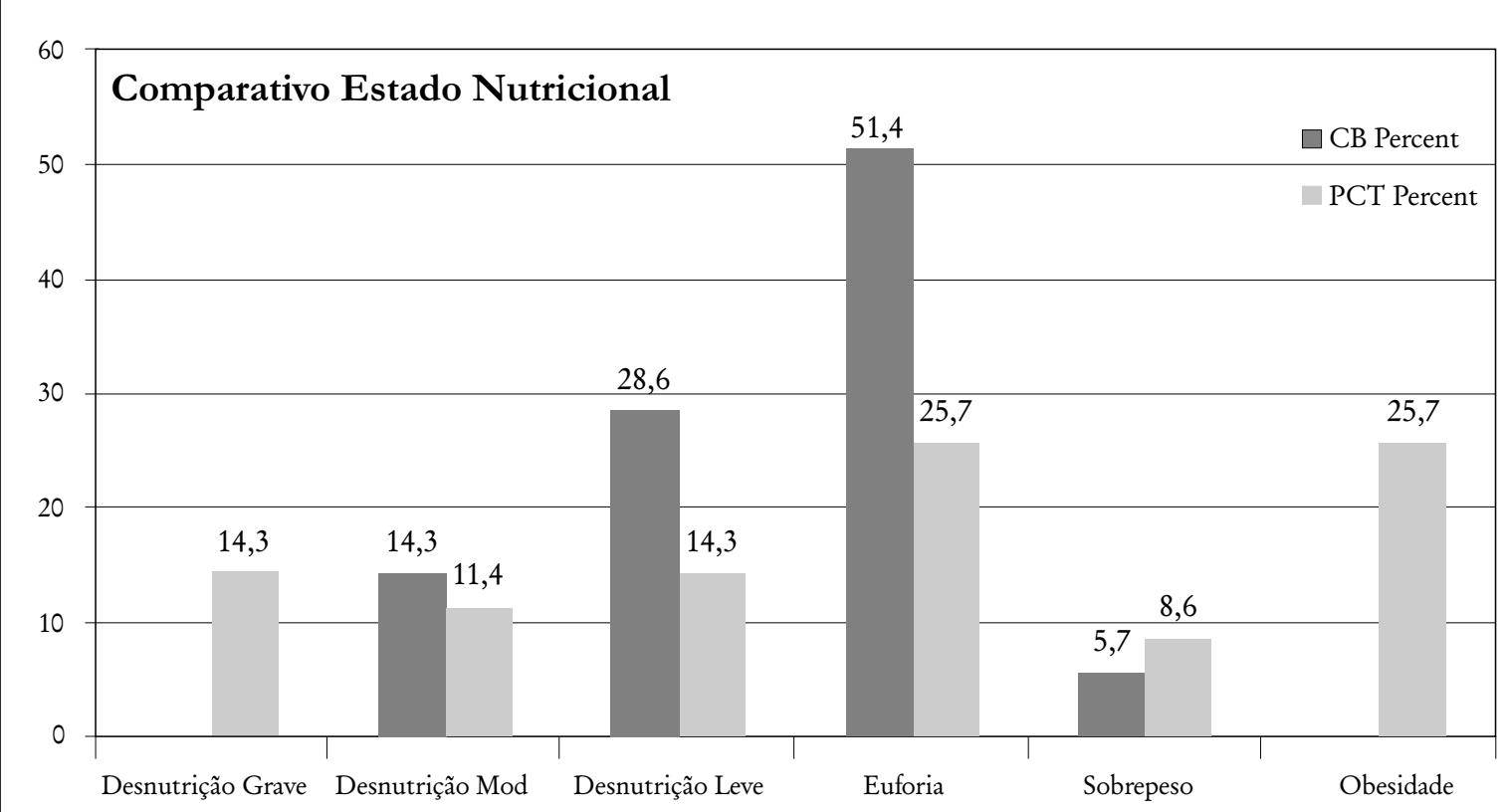

Figura 1 - Comparação da classificação do estado nutricional segundo a interpretação das variáveis antropométricas relacionadas à Prega Cutânea Tricipital e a Circunferência do Braço na amostra estudada. Curitiba (PR), 2009.

A análise da consistência interna (alfa de Cronbach) da aplicação do inquérito WHOQOL-bref na amostra foi de 0,927 para as 26 questões, e manteve-se superior a 0,5 na análise individual de cada um dos quatro domínios que compõem o instrumento. A pontuação média, consistência interna e questões que compõe os domínios físico, psicológico, relações e meio ambiente encontram-se na tabela 3 .

Tabela 3 - Caracterização das médias dos escores (0-100) dos domínios que compõe o Inquérito WHOQOL-bref na amostra de idosos institucionalizados. Curitiba, PR, 2009.

\begin{tabular}{lcccc}
\hline & alfa* & Questões & Média & $\begin{array}{c}\text { Desvio } \\
\text { Padrão }\end{array}$ \\
\hline Domínio 1 (físico) &, 833 & $Q 3, Q 4, Q 10, Q 15, Q 16, Q 17, Q 18$ & 60,81 & 20,72 \\
Domínio 2 (psicológico) &, 719 & $Q 5, Q 6, Q 7, Q 11, Q 19, Q 26$ & 58,92 & 17,91 \\
Domínio 3 (relações sociais) &, 625 & $Q 20, Q 21, Q 22$ & 62,14 & 18,88 \\
Domínio 4 (meio ambiente) &, 750 & $Q 8, Q 9, Q 12, Q 13, Q 14, Q 23, Q 24, Q 25$ & 56,33 & 15,87 \\
Q1 $†$ & NA & avaliação geral da qualidade de vida & 15,17 & 5,09 \\
Q2 $\dagger$ & NA & avaliação da satisfação com a saúde & 14,82 & 6,44 \\
\hline
\end{tabular}

* Alfa de Cronbach, medida de avaliação da consistência interna/confiabilidade do instrumento na amostra estudada. $\dagger$ Q1 e Q2 apresentados no escore 4-20.

$\doteqdot$ Não aplicável. 
A pontuação média dos domínios que compõem o WHOQOL-bref e as questões gerais de qualidade de vida (Q1 e Q2) foi maior para os idosos que apresentavam estado nutricional considerado como sobrepeso quando analisados segundo o IMC (Pearson; $\mathrm{p}=0,001$ ), mas a diferença na análise de variância (ANOVA) não foi significativa. Não houve diferença significativa também na análise de correlação e variância das percepções de QV segundo o EN pela CB.

A análise das percepções de QV segundo o $\mathrm{EN}$ conforme a PCT evidenciou significativa diferença nas médias dos domínios 3 e 4, destacando associação significativa entre ter sobrepeso e maiores médias (melhor percepção de QV) nos domínios citados (ANOVA; $\mathrm{p}=0,035$ e $\mathrm{p}=0,020$, respectivamente). Houve também evidente correlação entre melhores percepções e sobrepeso em todos os domínios (Pearson; $\mathrm{p}=0,013$ ).

A percepção de QV também foi analisada tomando em conta a variação das médias dos escores dos domínios e Q1 e Q2, segundo a distribuição por diferentes grupos etários. Quanto mais jovens eram os idosos analisados, maiores eram as pontuações de todos os domínios do WHOQOL-bref. Encontrou-se diferença significativa, porém, somente quando analisadas as médias do domínio $3(\mathrm{p}=0,029)$.

Tabela 4 - Caracterização das médias dos escores dos domínios mensurados pelo Inquérito WHOQOLbref de acordo com o estado nutricional segundo índice de massa corporal, prega cutânea tricipital e circunferência do braço na amostra de idosos institucionalizados. Curitiba, PR, 2009.

\begin{tabular}{ccccccc}
\hline & Dominio $1^{+}$ & Dominio $2^{+}$ & Dominio $3^{+}$ & Dominio $4^{*}$ & $\mathrm{Q}^{*}$ & $\mathrm{Q}^{*}$ \\
\hline IMC & & & & & & \\
$B P$ & 57,8571 & 51,3889 & 60,5556 & 52,0833 & 13,7500 & 15,4167 \\
$E U$ & 63,3929 & 63,8889 & 61,1111 & 58,0729 & 15,1042 & 13,0208 \\
$S B$ & 72,6190 & 68,0556 & 72,2222 & 64,5833 & 19,8333 & 18,7500 \\
$O B$ & 56,4286 & 64,1667 & 63,3333 & 60,0000 & 16,2500 & 15,0000 \\
& & & & & & \\
PCT & & & & & & \\
$D G$ & 63,5714 & 50,8333 & 66,6667 & 51,2500 & 13,7500 & 17,5000 \\
$D M$ & 73,2143 & 62,5000 & 45,8333 & 54,6875 & 12,5000 & 10,9375 \\
$D L$ & 50,7143 & 55,8333 & 45,0000 & 46,8750 & 13,7500 & 11,2500 \\
$E U$ & 53,1746 & 53,2407 & 66,6667 & 50,3472 & 14,5833 & 13,8889 \\
$S B$ & 86,9048 & 84,7222 & 80,5556 & 80,2083 & 19,8333 & 19,8333 \\
$O B$ & 58,3333 & 60,6481 & 65,7407 & 63,1944 & 16,6667 & 15,9722 \\
& & & & & & \\
CB & & & & & & \\
$D M$ & 70,0000 & 52,5000 & 63,3333 & 56,8750 & 13,7500 & 17,5000 \\
$D L$ & 61,4286 & 58,7500 & 68,3333 & 59,3750 & 16,2500 & 15,0000 \\
$E U$ & 58,9286 & 60,8796 & 59,7222 & 54,8611 & 15,2778 & 14,5833 \\
$S B$ & 51,7857 & 58,3333 & 50,0000 & 53,1250 & 12,5000 & 9,3750 \\
\hline
\end{tabular}

$\dagger$ Escore 0-100

$\ddagger$ Escore 4-20 
No entanto, a análise univariada para determinar se escores mais elevados de QV correlacionaram-se melhor com a idade ou EN não foi possível, pois não houve representantes em todas as categorias de EN em alguns dos métodos. Do mesmo modo, não foi encontrada associação entre as médias do tempo de institucionalização e o estado nutricional ou escores dos domínios do WHOQOL-bref.

\section{DISCUSSÃO}

Estudos têm estimado a prevalência de desnutrição na população idosa institucionalizada entre 15 a $20 \%$, podendo atingir cifras de até $60 \%$ da amostra pesquisada, de acordo com o método e características da amostra e estudo analisado. ${ }^{19,20}$

Em nossa amostra de homens idosos institucionalizados, encontramos uma prevalência de baixo-peso segundo o IMC de $48,8 \%$ na amostra geral $(n=35)$. Ao analisar o estado nutricional nos três diferentes segmentos etários, ao contrário do que se poderia esperar, o grupo etário de 80 anos ou mais foi o que mostrou menor prevalência de baixo-peso $(12,5 \% ; n=8)$.

Quando o estado nutricional foi analisado segundo a interpretação da PCT e CB, a prevalência de desnutrição na amostra total variou entre $11,4 \%$ e $28,6 \%$, enquanto a prevalência de eutrofia (que era de $34,3 \%$, segundo o IMC), variou de $25,7 \%$ e $51,4 \%$, respectivamente, segundo a PCT e CB.

Sabidamente, o estado nutricional do idoso pode variar significativamente conforme o tipo de domicilio aonde reside o idoso. Estudos realizados com idosos residentes na comunidade mostram anciões com EN predominantemente eutróficos. ${ }^{2}$ Além da questão do tipo de moradia, o gênero também pode influenciar sobremaneira os resultados de inquéritos antropométricos neste segmento etário. Em Fortaleza, Menezes \& Marucci ${ }^{4}$ encontraram que os homens institucionalizados apresentaram estatura média superior à das mulheres, e valor médio de peso significativamente superior em homens quando comparados às mulheres.

Em inquérito realizado em instituição mexicana de longa permanência para idosos, utilizando-se o protocolo de Mini-Avaliação Nutricional $^{\S}$ (MAN), Álamo e colaboradores ${ }^{19}$ encontraram prevalência de desnutrição de $6 \% \mathrm{em}$ amostra de 55 residentes, mas $12 \%$ adicionais apresentavam risco de desnutrição, enquanto $44 \%$ da população estudada apresentava obesidade, valor superior a outros estudos, incluindo os achados de nossa população, onde a prevalência de obesidade variou de 14,3\% (segundo o IMC) a 25,7\% (segundo a PCT).

Rauen et al, ${ }^{21}$ estudando idosos de ambos os gêneros, encontraram valores de IMC que categorizaram $45,5 \%$ da amostra em baixo-peso, $33,5 \%$ de individuos eutróficos, $7,8 \%$ de préobesos e 13,2\% de obesos. Melo ${ }^{22}$ encontrou resultados semelhantes quando analisou o $\mathrm{EN}$ pelo IMC, identificando que para homens idosos prevalecem a desnutrição $(70,3 \%)$ e eutrofia $(18,5 \%)$, ou seja, a maioria da amostra entre os baixo-pesos e eutróficos. No mesmo estudo, ao comparar o EN segundo a PCT, resultados distintos foram encontrados, pois neste $55,5 \%$ tinham peso insuficiente, $33,3 \%$ eram eutróficos e $11,1 \%$ apresentam-se com peso excessivo.

A pesquisa e a produção científica envolvendo a temática da qualidade de vida $(\mathrm{QV})$ tiveram crescimento expressivo nas duas últimas décadas, em especial nos últimos cinco anos. De fato, análises empíricas sobre quais fatores influenciariam positiva ou negativamente a experiência individual ou coletiva do envelhecer vêm sendo rapidamente abandonadas, à medida que instrumentos e pesquisas exploratórias e ensaios clínicos conseguem efetivamente comprovar o impacto significativo que mudanças nestas percepções de QV mantêm com desfechos em saúde e doença.

Estudo publicado por Xavier et al. ${ }^{23}$ destaca que $57 \%$ da amostra de idosos estudados por esses autores definiam sua atual qualidade de vida como positiva. Os sujeitos que se declaram insatisfeitos 
com suas QV tinham mais problemas de saúde e mais sintomas depressivos, além de se preocuparem mais com seu status econômico, quando comparados com os idosos que se declararam satisfeitos. A religiosidade, segundo o mesmo estudo, não pareceu influenciar percepções mais positivas ou negativas.

Analisando fatores associados à qualidade de vida em idosos ativos, Alexandre, Cordeiro \& Ramos $^{24}$ encontraram que não ter vida conjugal, ter maior escolaridade e renda mensal superior ao equivalente a U\$ 687,76 favoreciam percepções positivas da vida neste segmento etário, em amostra com características específicas clássicas de envelhecimento bem-sucedido.

Estudo publicado por Joia et al., ${ }^{25}$ realizado com 375 idosos residentes na comunidade, no município de Botucatu(SP), evidenciou que $51,5 \%$ dos entrevistados se disseram satisfeitos com a vida em geral e 43,6\% declararam-se muito satisfeitos. Os autores conseguiram encontrar associação de melhor satisfação com a vida com conforto domiciliar, não sentir-se só, acordar bem pela manhã, não ser diabético, ter atividades de lazer e, interessantemente, ter três ou mais refeições diárias.

Hickson \& Frost, ${ }^{26}$ ao pesquisar em idosos londrinos a relação entre qualidade de vida, estado nutricional e função física, concluíram que o estado nutricional não influencia a qualidade de vida, mas poderia afetar diretamente a função física. Digno de nota, porém, é o fato de que o estado nutricional, nesse estudo, limitava-se à avaliação do número de refeições diárias, o que agrega significativo viés à interpretação dessas conclusões.

Almeida \& Rodrigues, ${ }^{27}$ pesquisando idosos portugueses institucionalizados, também encontraram elevadas taxas de satisfação com sua qualidade de vida (51,6\% da amostra total de 93 idosos). Digno de nota foi a diferença significativa observada entre os gêneros, com muito mais homens declarando melhores percepções de QV, e o fato de que quanto maior foi a idade do entrevistado, piores eram as avaliações.
Nossos dados identificaram melhores percepções de qualidade de vida quando analisadas as médias dos escores dos domínios e questões gerais que compõem o instrumento WHOQOL-bref nos homens idosos com sobrepeso segundo o IMC. A mesma significância, porém, não foi encontrada quando se analisaram as médias dos domínios e questões gerais segundo o EN pela CB.

Associações significativas foram encontradas para os domínios 3 e 4 (relações sociais e meio ambiente, respectivamente) nos indivíduos com sobrepeso conforme a PCT, além de maiores escores em todos os domínios, quando comparados com os idosos desnutridos, eutróficos e obesos. À semelhança dos achados de Almeida \& Rodrigues, ${ }^{27}$ quanto mais jovens eram os idosos da amostra, maiores eram as médias em todos os domínios e também nas questões gerais que avaliaram a qualidade de vida nessa população.

Em acordo com Chachamovich, ${ }^{28}$ acreditamos que as intervenções em idosos devam possibilitar a manutenção da mobilidade, independência, nível de atividade, participação social e capacidade de responder adequadamente às demandas físicas e psicológicas da velhice. De modo a poder atingir estes objetivos, é impar que se reconheçam quais fatores efetivamente se correlacionam e propiciam qualidade de vida aos anos de vida restantes. Poucos estudos têm, até o momento, se preocupado com a importância e o impacto do estado nutricional nas percepções de qualidade de vida dos idosos, institucionalizados ou não.

Este estudo tem como limitações não ter analisado o impacto da presença de comorbidades, o uso de polifármacia, e outras variáveis na análise da percepção de melhor ou pior qualidade de vida, segundo os escores do WHOQOL-bref. As características da população estudada e a reduzida amostra, do mesmo modo, limitam a extrapolação dos achados desta pesquisa.

Os resultados deste estudo, porém, reacendem a importância da discussão e melhor exploração dos dados encontrados em análises antropométricas, como o estado nutricional, e 
seu potencial impacto como um dos fatores a contribuir com percepções mais ou menos positivas ou negativas de qualidade de vida na velhice. E reforçam o imaginário comum (não necessariamente ainda cientificamente comprovado ou descartado) de que idosos com sobrepeso têm mais reserva para atingir idades longevas, e sentem-se fisicamente mais fortes (ou aptos a realizar as atividades necessárias à vida diária) e, portanto, mais satisfeitos com sua vida.

\section{CONCLUSÃO}

A percepção de qualidade de vida na amostra foi melhor para os idosos de menor idade, independentemente do estado nutricional. Quanto maior a idade, menores foram as médias dos escores em todos os domínios do WHOQOL-bref.

Analisando o EN através de três métodos, encontramos interpretações diferenciadas de acordo com o método utilizado: de acordo com

\section{REFERÊNCIAS}

1. Garcia ANM, Romani SAM, Lira PIC. Indicadores Antropométricos em idosos. Rev. Nutr. 2007; 20(4): 371-378.

2. Moreira AJ, Nicastro H, Cordeiro RC, Coimbra P, Frangella VS. Composição corporal de idosos segundo a antropometria. Rev.Bras.Geriatr.Gerontol 2009; 12(2): 201-213.

3. Busnello FM. Aspectos nutricionais no processo do envelhecimento. São Paulo: Atheneu; 2007.

4. Menezes TN, Marucci MFN. Antropometria de idosos residentes em instituições geriátricas de Fortaleza-CE. Rev Saúde Pública 2005; 39(2): 169-75.

5. Garcia SS, et al. Anthropometric measures and nutritional status in a healthy elderly population. BMC Public Health 2007; 7(2):1-9.

6. Ramos LJ. Avaliação do estado nutricional de seis domínios da qualidade de vida e da capacidade de tomar decisão de idosos institucionalizados e não-institucionalizados no município de Porto Alegre Rio Grande do sul. Dissertação [mestrado em Nutrição]- o IMC, os idosos com sobrepeso apresentam melhor percepção de QV. O EN pela CB indicou que os idosos eutróficos demonstram melhor percepção de QV, mas também não de modo significativo. O EN encontrado através PCT indicou que idosos com sobrepeso detêm as melhores percepções de QV, principalmente associadas com as questões que envolvem os domínios relações sociais e meio ambiente, o que é condizente com a vida em instituição.

Destaca-se a grande importância da nutrição para a qualidade de vida do idoso nessa instituição, pois tanto pelo IMC quanto pela PCT, a desnutrição e a obesidade revelaram-se indicadores de insatisfação para a qualidade de vida dessa população, quando comparados com seus pares eutróficos ou com sobrepeso.

\section{AGRADECIMENTOS}

Agradecemos à Professora Carmem Luciane Sanson Abourihan e às Faculdades Integradas do Brasil, pelo apoio institucional.
Pontifícia Universidade Católica do Rio Grande do Sul; 2008.

7. Sonati JG. Caracterização da transição da idade adulta para a terceira idade quanto ao estado nutricional, composição corporal e percepção da qualidade de vida. Campinas.Dissertação [Mestrado em nutrição] - Universidade Estadual de Campinas; 2007.

8. Navarro MLBA, Bennemann RM. Avaliação do estado nutricional de idosos residentes em uma instituição asilar da cidade de MarialvaPR. Acta Sci. Health Sci. 2006; 28(2):129-135.

9. Vecchia RD. Qualidade de vida na terceira idade: um conceito subjetivo. Rev. Bras. Epidemiol 2005; 8(3):246-52.

10. Sousa L, Galante H, Figueiredo D. Qualidade de vida dos idosos. Rev Saúde Pública 2003; 37(3): 364-71.

11. Fleck MPA. O instrumento de avaliação de qualidade de vida da Organização Mundial da Saúde: características e perspectivas. Ciênc Saúd Colet 2000; 5(1):33-38. 
12. Metter EJ. Skeletal muscle strengh as a predictor of all cause mortality in healthy men.J Gerontol 2002; 57(10):359-365.

13. Chumlea WC, Roche AF, Steinbaugh ML. Estimating stature from knee height for persons 60 to 90 years of age. J Am Geriatr Soc 1985; $33: 116-120$.

14. Chumlea WC, Guo S, Roche AF, Steinbaugh ML. Prediction of body weight for the nonambulatory elderly from anthropometry. J Am Diet Assoc 1988; 88:564-568.

15. Blackburn GL, Thornton PA. Nutrition assessment of the hospitalized patients. Med Clin North Am 1979;63:1103-15.

16. Frisancho AR. New norms of upper limb fat and muscle areas for assessment of nutritional status. Am J Clin Nutr 1981; 34(11): 2540-2545.

17. World Health Organization. Sintaxe SPSS: WHOQOL-bref Questionnaire. [Acesso em out 2009 ]. Disponível em < http://www.ufrgs.br/ psiq/whoqol86.html $>$.

18. Almeida OP. Mini exame do estado mental e o diagnóstico de demência no Brasil. Arq Neuropsiquiatr. 1998; 56(3-B):605-612.

19. Álamo AC,et al. Protocolo de valoración, seguimiento y actuación nutricional en un residencial para personas mayores. Nutr Hosp 2008; 23(2): 100-104.

20. Estebán M, Fernandéz J, Salvado J. Estado nutricional de la población anciana em función del regimen de institucionalización. Nutr. Hosp.2000; 3:64-72.
21. Rauen MS, Moreira EAM, Calvo MCM, Lobo AS. Avaliação do estado nutricional de idosos institucionalizados. Rev. Nutr 2008; 21(3):303-310.

22. Melo MTS. Avaliação do estado nutricional de idosos residentes em Instituições de longa permanência em Teresina.Teresina. Dissertação [Mestrado em Nutrição]-Universidade Federal do Piauí; 2008.

23. Xavier FMF, et al. Elderly people's definition of quality of life. Rev.Bras.Psiquiatr 2003; 25(1):31-39.

24. Alexandre TS, Cordeiro RC, Ramos LR. Factors associated to the quality of life in active elderly. Rev. Saúde Pública 2009; 43(4): 613-621.

25. Joia LC, Ruiz T, Donalisio MR. Condições associadas ao grau de satisfação com a vida entre população de idosos. Rev. Saúde Públ. 2007; 41(1): 131-138.

26. Hickson M, Frost G. A investigation into the relationships between quality of life, nutritional status and physical function. Clin Nutr 2004; 23(2):213-21.

27. Almeida AJS, Rodrigues VMCP. The quality of life of aged people living in homes for the aged. Rev. Latino-am. Enfermagem 2008; 16(6): 1025-1031.

28. Chachamovich E. Qualidade de vida em idosos: desenvolvimento e aplicação do módulo WHOQOL-Old e teste do desempenho do instrumento WHOQOL-bref em uma amostra de idosos brasileiros. Porto Alegre. Dissertação [Mestrado em Nutrição]— Universidade Federal do Rio Grande do Sul; 2005. 
